\title{
ON THE INITIAL-BOUNDARY VALUE PROBLEMS FOR A DEGENERATE PARABOLIC EQUATION
}

\author{
JAN GONCERZEWICZ \\ Institute of Mathematics and Computer Science, Wrockaw University of Technology \\ Janiszewskiego 14a, 50-372 Wrockaw, Poland \\ E-mail: goncerz@im.pwr.wroc.pl
}

\begin{abstract}
For an equation of the type of porous media equation the Cauchy-Dirichlet and Cauchy-Neumann problems are considered. The existence and uniqueness results in the case of $L^{\infty}$ initial and boundary data are given.
\end{abstract}

\section{Introduction. Let}

$$
Q_{T}:=(1, \infty) \times(0, T]
$$

For the equation

$$
u_{t}=r^{-d}\left(r^{d}\left(u^{m}\right)_{r}\right)_{r}
$$

where $m>1$ and $d$ are real parameters we consider the following two initial-boundary value problems: the Cauchy-Dirichlet problem

$$
(\mathrm{CD}) \begin{cases}u_{t}=r^{-d}\left(r^{d}\left(u^{m}\right)_{r}\right)_{r} & \text { for }(r, t) \in Q_{T}, \\ u(1, t)=f(t) & \text { for } t \in(0, T), \\ u(r, 0)=u_{0}(r) & \text { for } r \in(1, \infty),\end{cases}
$$

and the Cauchy-Neumann problem

$$
(\mathrm{CN}) \begin{cases}u_{t}=r^{-d}\left(r^{d}\left(u^{m}\right)_{r}\right)_{r} & \text { for }(r, t) \in Q_{T}, \\ -\left(u^{m}\right)_{r}(1, t)=g(t) & \text { for } t \in(0, T), \\ u(r, 0)=u_{0}(r) & \text { for } r \in(1, \infty) .\end{cases}
$$

Throughout the whole paper we assume that

(A) $u_{0}$ is a bounded, nonnegative, measurable function on $(1, \infty)$, and, $f$ and $g$ are bounded, nonnegative, measurable functions on $(0, T)$.

2000 Mathematics Subject Classification: Primary 35K65; Secondary 35K60.

Key words and phrases: degenerate parabolic equation, porous media equation, CauchyDirichlet problem, Cauchy-Neumann problem, weak solution.

The paper is in final form and no version of it will be published elsewhere. 
If $d=N-1$ and $N>1$ is a positive integer then equation (1.1) is the radial version of the porous media equation (PME)

$$
u_{t}=\Delta u^{m}
$$

in which $\Delta$ is the Laplace operator in $\mathbb{R}^{N}$ and $t$ denotes the time variable. In this case the problems under consideration correspond to the exterior-domain problems with radial symmetry in which the space domain comprises the complement of the unit ball in $\mathbb{R}^{N}$ and the Dirichlet or Neumann data are prescribed on the boundary of the corresponding space-time domain. If $N=1$ we get to the problems posed in a quarter plane for onedimensional PME. Note that analogous problems posed in $(\rho, \infty) \times(0, T]$ with arbitrary $\rho>0$ can be converted into the problems in $Q_{T}$ by scaling $\widetilde{u}(r, t):=\rho^{2 /(m-1)} u(r / \rho, t)$ (cf [8]).

There exists a vast literature devoted to PME: we refer to [19] (the most recent) or [2, $12,16,18$ ] (earlier ones) and the literature cited therein. When $N=1$ basic existence and uniqueness results concerning above Cauchy-Dirichlet and Cauchy-Neumann problems can be found in [14], however, under assumptions that the initial and boundary data functions $u_{0}, f$ and $g$ are sufficiently smooth and satisfy suitable compatibility conditions. These regularity assumptions on the data became typical in the literature devoted to more general (degenerate) diffusion-convection equations ([3], [6], [7],...). Analogous theory in the case $N>1$ and unbounded space domain is less complete: some results in a framework of $L^{1}$ theory for the Cauchy-Dirichlet problem and for the Cauchy-Neumann problem with homogeneous boundary data are available [18, 19].

The aim of this paper is to present the existence and uniqueness theory for Problems $\mathrm{CD}$ and $\mathrm{CN}$ for $L^{\infty}$ initial and boundary data. We also admit $d$ to be any real number. The results gathered here complete details of the basic theory needed for [8] and [9], where certain properties of solutions of the above problems are considered. Since the proofs of the results for both problems are similar we only give the details for Problem CN (which needs some less standard arguments than CD).

Because of the type of the equation (degenerate parabolic) we shall work with the weak solutions of the problems.

Definition 1. A function $u$ defined on $Q_{T}$ is said to be a weak solution of Problem CD if $u$ is nonnegative, bounded and continuous in $Q_{T}$ and $u$ satisfies the identity

$$
\begin{aligned}
& \int_{0}^{T} \int_{1}^{\infty} r^{d}\left\{u \xi_{t}+u^{m} r^{-d}\left(r^{d} \xi_{r}\right)_{r}\right\} d r d t \\
& \quad+\int_{1}^{\infty} r^{d} u_{0}(r) \xi(r, 0) d r+\int_{0}^{T} f^{m}(t) \xi_{r}(1, t) d t=0
\end{aligned}
$$

for all nonnegative $\xi \in C^{2,1}\left(\overline{Q_{T}}\right)$ which vanish for $t=T, r=1$, and large $r$. If in (1.2) the equality is replaced by $\leq(\geq)$ then we call $u$ a weak supersolution (subsolution) of Problem CD.

Definition 2. A function $u$ defined on $Q_{T}$ is said to be a weak solution of Problem CN if $u$ is nonnegative, bounded and continuous in $Q_{T}$ and $u$ satisfies the identity 


$$
\begin{aligned}
& \int_{0}^{T} \int_{1}^{\infty} r^{d}\left\{u \xi_{t}+u^{m} r^{-d}\left(r^{d} \xi_{r}\right)_{r}\right\} d r d t \\
& \quad+\int_{1}^{\infty} r^{d} u_{0}(r) \xi(r, 0) d r+\int_{0}^{T} g(t) \xi(1, t) d t=0
\end{aligned}
$$

for all nonnegative $\xi \in C^{2,1}\left(\overline{Q_{T}}\right)$ which vanish for $t=T$ and large $r$, and are such that $\xi_{r}$ vanishes for $r=1$. If in (1.3) the equality is replaced by $\leq(\geq)$ then we call $u$ a weak supersolution (subsolution) of Problem CN.

\section{Comparison and uniqueness results}

2.1. Cauchy-Neumann problem. We begin with the following lemma in which we give an equivalent definition of the solution (resp. super- and subsolution) of Problem CN.

LEMma 1. A function $u$ is a weak solution of Problem $C N$ if and only if $u$ is nonnegative, bounded and continuous in $Q_{T}$ and satisfies instead of (1.3) the identity

$$
\begin{aligned}
& \iint_{P} r^{d}\left\{u \xi_{t}+u^{m} r^{-d}\left(r^{d} \xi_{r}\right)_{r}\right\} d r d t \\
& +\int_{1}^{R} r^{d} u_{0}(r) \xi(r, 0) d r+\int_{0}^{T} g(t) \xi(1, t) d t-\left.\int_{0}^{T} r^{d} u^{m} \xi_{r} d t\right|_{r=R}=0
\end{aligned}
$$

for all rectangles $P \equiv[1, R] \times[0, T]$ with $1<R<\infty$ and for all nonnegative $\xi \in C^{2,1}(P)$ which vanish for $t=T$ and $r=R$ and are such that $\xi_{r}(1, t)=0$ for $t \in[0, T]$. Similarly, defining a weak supersolution (resp. subsolution) of Problem $C N$ one should replace the equality in (2.1) by $\leq$ (resp. $\geq$ ).

The proof of this lemma goes analogously to the proof of Theorem 3.2 in [3] and we omit the details.

We prove the following theorem.

THEOREM 1. Let $u$ be a weak solution of Problem $C N$ with the initial and boundary data functions $u_{0}, g$, and let $\bar{u}$ (resp. $\underline{u}$ ) be a weak supersolution (resp. subsolution) of Problem $C N$ with the initial and boundary data functions $\bar{u}_{0}, \bar{g}$ (resp. $\underline{u}_{0}, \underline{g}$ ) which satisfy assumption $(A)$. For any nonnegative $\chi \in C_{0}^{\infty}\left(Q_{T}\right)$ there exist positive constants $C_{1}$ and $C_{2}$ such that

$$
\begin{aligned}
\iint_{Q_{T}} r^{d}(u-\bar{u}) \chi d r d t \leq & C_{1} \int_{1}^{\infty} r^{d} e^{-r}\left(u_{0}(r)-\bar{u}_{0}(r)\right)^{+} d r \\
& +C_{2} \int_{0}^{T}(g(t)-\bar{g}(t))^{+} d t \\
\left(\text { resp. } \iint_{Q_{T}} r^{d}(u-\underline{u}) \chi d r d t \leq\right. & C_{1} \int_{1}^{\infty} r^{d} e^{-r}\left(u_{0}(r)-\underline{u}_{0}(r)\right)^{+} d r \\
& \left.+C_{2} \int_{0}^{T}(g(t)-\underline{g}(t))^{+} d t\right) .
\end{aligned}
$$

Proof. We shall only prove the corresponding inequality for $u$ and $\bar{u}$. In the proof we follow the ideas of [11]. 
Let $\chi \in C_{0}^{\infty}\left(Q_{T}\right)$ be a nonnegative function and let $\bar{r}$ be a real number such that $\chi=0$ for all $r \geq \bar{r}$. Let $R \geq \bar{r}+1$ and denote by $P$ the rectangle $[1, R] \times[0, T]$. In view of Lemma 1 for all nonnegative $\xi \in C^{2,1}(P)$, and such that $\left.\xi_{r}\right|_{r=1}=\left.\xi\right|_{r=R}=\left.\xi\right|_{t=T}=0$ we have

$$
\begin{aligned}
\iint_{P} r^{d}(\bar{u}-u)\left\{\xi_{t}\right. & \left.+a(r, t) r^{-d}\left(r^{d} \xi_{r}\right)_{r}\right\} d r d t+\int_{1}^{R} r^{d}\left(\bar{u}_{0}-u_{0}\right) \xi(r, 0) d r \\
+\int_{0}^{T}(\bar{g}(t)-g(t)) \xi(1, t) d t-\left.\int_{0}^{T} r^{d}\left(\bar{u}^{m}-u^{m}\right) \xi_{r} d t\right|_{r=R} \leq 0 & \leq
\end{aligned}
$$

where

$$
a=a(r, t)= \begin{cases}\frac{\bar{u}^{m}-u^{m}}{\bar{u}-u} & \text { for } \bar{u} \neq u, \\ 0 & \text { otherwise. }\end{cases}
$$

Observe that $a$ is bounded and nonnegative on $Q_{T}$. Let $\left\{a_{n}\right\}_{n=1}^{\infty} \subset C^{\infty}\left(Q_{T}\right)$ be a sequence of functions of the form

$$
a_{n}=\widetilde{a} * \rho_{n}+1 / n,
$$

where $\widetilde{a}$ is the extension by 0 of $a$ to the whole $\mathbb{R}^{2}$ and $\left\{\rho_{n}\right\}_{n=1}^{\infty}$ is a sequence of mollifiers in $\mathbb{R}^{2}$ such that

$$
\iint_{P}\left(a-\widetilde{a} * \rho_{n}\right)^{2} d r d t \leq \frac{1}{n^{2}}
$$

Then

$$
\frac{1}{n} \leq a_{n} \leq\|a\|_{\infty}+1=M
$$

and,

$$
\left(\iint_{P} \frac{\left(a-a_{n}\right)^{2}}{a_{n}} d r d t\right)^{1 / 2} \leq \frac{1+\sqrt{R T}}{\sqrt{n}} .
$$

Substituting $a=a_{n}+\left(a-a_{n}\right)$ into (2.4) yields

$$
\begin{aligned}
\iint_{P} r^{d}(\bar{u}-u)\left\{\xi_{t}+a_{n} r^{-d}\left(r^{d} \xi_{r}\right)_{r}\right\} d r d t & \\
+\iint_{P}(\bar{u}-u)\left(a-a_{n}\right)\left(r^{d} \xi_{r}\right)_{r} d r d t+\int_{1}^{R} r^{d}\left(\bar{u}_{0}-u_{0}\right) \xi(r, 0) d r & \\
& +\int_{0}^{T}(\bar{g}(t)-g(t)) \xi(1, t) d t-\left.\int_{0}^{T} r^{d}\left(\bar{u}^{m}-u^{m}\right) \xi_{r} d t\right|_{r=R} \leq 0
\end{aligned}
$$

for all $\xi \in C^{2,1}(P), \xi \geq 0$ and such that $\left.\xi_{r}\right|_{r=1}=\left.\xi\right|_{r=R}=\left.\xi\right|_{t=T}=0$.

For any fixed $n$ and $R>r_{0}$ consider now the following backward parabolic problem

$$
\left\{\begin{array}{l}
\xi_{t}+a_{n} r^{-d}\left(r^{d} \xi_{r}\right)_{r}=-\chi \quad \text { in } P, \\
\left.\xi_{r}\right|_{r=1}=\left.\xi\right|_{r=R}=\left.\xi\right|_{t=T}=0 .
\end{array}\right.
$$

It follows from $[4,13]$ that problem $(2.8)-(2.9)$ has a unique solution $\xi^{n, R} \in C^{2,1}(P)$. We state some useful properties of $\xi^{n, r}$ in the following lemma. 
Lemma 2. There are positive constants $C_{1}, C_{2}$ and $C_{3}$, that do not depend on $n$ and $R$, and such that

$$
\begin{gathered}
0 \leq \xi^{n, R}(r, t) \leq C_{1} e^{-r} \quad \text { for }(r, t) \in P, \\
-C_{2} e^{-R} \leq \xi_{r}^{n, R}(R, t) \leq 0 \quad \text { for } t \in[0, T], \\
\iint_{P} a_{n}\left\{\left(r^{d} \xi_{r}^{n, R}\right)_{r}\right\}^{2} d r d t \leq C_{3} .
\end{gathered}
$$

We postpone the proof of this lemma until the end of this section. Now we continue the proof of Theorem 1. After substituting $\xi^{n, R}$ instead of $\xi$ into (2.7) we obtain

$$
\begin{aligned}
& \iint_{P} r^{d}(u-\bar{u}) \chi d r d t+I(n, R)+\int_{1}^{R} r^{d}\left(\bar{u}_{0}-u_{0}\right) \xi^{n, R}(r, 0) d r \\
&+\int_{0}^{T}(\bar{g}(t)-g(t)) \xi^{n, R}(1, t) d t-\left.\int_{0}^{T} r^{d}\left(\bar{u}^{m}-u^{m}\right) \xi_{r}^{n, R} d t\right|_{r=R} \leq 0
\end{aligned}
$$

where we set

$$
I(n, R)=\iint_{P}(\bar{u}-u)\left(a-a_{n}\right)\left(r^{d} \xi_{r}^{n, R}\right)_{r} d r d t .
$$

Applying (2.10)-(2.11) of Lemma 2 yields

$$
\begin{aligned}
& \iint_{P}(u-\bar{u}) \chi d r d t \leq|I(n, r)|+C_{1} \int_{0}^{R} r^{d} e^{-r}\left(u_{0}(r)-\bar{u}_{0}(r)\right)^{+} d r \\
&+C_{1} \int_{0}^{T}(g(t)-\bar{g}(t))^{+} d t+\left.C_{2} R^{d} e^{-R} \int_{0}^{T}\left|\bar{u}^{m}-u^{m}\right| d t\right|_{x=R} .
\end{aligned}
$$

In view of $(2.12)$

$$
|I(n, R)| \leq C_{4}^{1 / 2}\left\{\iint_{P}(\bar{u}-u)^{2} \frac{\left(a-a_{n}\right)^{2}}{a_{n}} d r d t\right\}^{1 / 2}
$$

Hence, by (2.6),

$$
I(n, R) \rightarrow 0 \quad \text { as } n \rightarrow \infty
$$

for any fixed $R \geq r_{0}+1$. Taking limits in (2.13), first when $n \rightarrow \infty$ and then when $R \rightarrow \infty$ we obtain $(2.2)$.

Corollary 1. Let $u$ and $\bar{u}$ (resp. $\underline{u}$ ) be as in Theorem 1. If $u_{0} \leq \bar{u}_{0}$ (resp. $\underline{u}_{0} \leq u_{0}$ ) a.e. in $(1, \infty)$ and $g \leq \bar{g}$ (resp. $g \leq g$ ) a.e. in $(0, T)$ then $u \leq \bar{u}$ (resp. $\underline{u} \leq u$ ) in $S_{T}$.

Proof. The assertion follows since we have

$$
\iint_{Q_{T}}(u-\bar{u}) \chi d r d t \leq 0 \quad\left(\operatorname{resp} . \quad \iint_{Q_{T}}(\underline{u}-u) \chi d r d t \leq 0\right)
$$

for any nonnegative $\chi \in C_{0}^{\infty}\left(Q_{T}\right)$.

Corollary 2. Let $u$ and $\tilde{u}$ be the weak solutions of Problem $C N$ with the data $u_{0}, g$ and $\tilde{u}_{0}, \tilde{g}$ respectively. If $u_{0} \leq \tilde{u}_{0}$ a.e. in $(1, \infty)$ and $g \leq \tilde{g}$ a.e. in $(0, T)$ then $u \leq \tilde{u}$ in $Q_{T}$.

Proof. The assertion follows from Corollary 1 by taking $\bar{u}=\tilde{u}$. 
Corollary 3. The weak solution of Problem $C N$ is unique.

Proof. This is an obvious consequence of Corollary 2.

Proof of Lemma 2. We follow the maximum principle technique used in [11]. In the sequel for the notational convenience we drop superscripts $n$ and $R$ in $\xi^{n, R}$. Let us denote

$$
\mathcal{L} \xi \equiv \xi_{t}+a_{n} r^{-d}\left(r^{d} \xi_{r}\right)_{r}
$$

and let $\Gamma$ denote the parabolic boundary of $P$ with respect to the differential operator $\mathcal{L}$.

We first prove that $\xi \geq 0$ in $P$. Arguing by contrary, suppose that $\xi$ attains its negative minimum at some point $\left(r_{0}, t_{0}\right) \in P$. Clearly it cannot happen when $t_{0}=T$ nor $r_{0}=R$. If it happens when $r_{0}=1$ then by the boundary minimum principle [17], $\xi_{r}\left(r_{0}, t_{0}\right)>0$ which violates the boundary condition at $r=r_{0}$. Finally if $\left(r_{0}, t_{0}\right) \in P \backslash \Gamma$ then by the strong maximum principle $\xi \equiv \xi\left(r_{0}, t_{0}\right)$ for all $t_{0} \leq t \leq T$ which contradicts $(2.9)$.

To prove the right hand side inequality in (2.10) we compare $\xi$ with the auxiliary function

$$
z(r, t)=\exp \{2 \kappa(T-t)+(\bar{r}-r)\}
$$

for $(r, t) \in P$, where $\kappa$ is a positive parameter to be chosen. Since $\left.(z-\xi)\right|_{t=T}>0$, $\left.(z-\xi)\right|_{r=R}>0$ and $\left.(z-\xi)_{r}\right|_{r=1}<0$ then $z-\xi$ cannot attain the negative minimum on $\Gamma$. We have

$$
\mathcal{L} z=z\left[-2 \kappa+a_{n}(1-(d / r)]\right) \leq-\kappa z<0
$$

for $\kappa$ sufficiently large $(\kappa>M(1+|d|)$ with $M$ given by $(2.5))$. Then $\mathcal{L}(z-\xi) \leq-\kappa z+\chi<$ 0 , again, for $\kappa$ large enough $\left(\kappa>\max _{Q_{T}} \chi\right)$. Therefore $z-\xi$ cannot attain the negative minimum in $P \backslash \Gamma$. Then $z-\xi \geq 0$ gives $(2.10)$ with $C_{1}=\exp \{2 \kappa T+\bar{r}\}$.

For the proof of (2.11) observe that $\xi \geq 0$ in $P$ and $\left.\xi\right|_{r=R}=0$ implies $\left.\xi_{r}\right|_{r=R} \leq 0$. To prove left hand side inequality we compare $\xi$ with

$$
\tilde{z}(r, t)=2 C_{1} \exp \{-(R-1)\}(r-R)
$$

in $\widetilde{P}=[R-1, R] \times[0, T]$. We have $\tilde{z}+\xi \leq 0$ on the parabolic boundary of $\widetilde{P}$ (again taken with respect to $\mathcal{L})$ and $\mathcal{L}(\tilde{z}+\xi)>0$ in $\widetilde{P}$. Then, by the maximum principle, $\tilde{z}+\xi \leq 0$ in $\widetilde{P}$. Since $\left.(\tilde{z}+\xi)\right|_{r=R}=0$ then $\left.(\tilde{z}+\xi)_{r}\right|_{r=R} \geq 0$ and (2.11) follows.

In order to prove (2.12) we multiply equation (2.8) by $r^{d}\left(r^{d} \xi_{r}\right)_{r}$ and integrate over $P$. Performing integration by parts yields

$$
\left.(1 / 2) \int_{1}^{R}\left(r^{d} \xi_{r}\right)^{2}\right|_{t=0} d r+\iint_{P} a_{n}\left\{\left(r^{d} \xi_{r}\right)_{r}\right\}^{2} d r d t=-\iint_{P}\left[r^{d}\left(r^{d} \chi_{r}\right)\right]_{r} \xi d r d t .
$$

By (2.10), the right hand side term of the above equality is bounded uniformly with respect to $n$ and $R$. Then the result follows.

2.2. Cauchy-Dirichlet problem. In this section we state comparison and uniqueness results for Problem CD. The counterpart of Theorem 1 is now the following.

TheOREm 2. Let $u$ be a weak solution of Problem CD with the initial and boundary data functions $u_{0}, f$, and let $\bar{u}$ (resp. $\underline{u}$ ) be a weak supersolution (resp. subsolution) of Problem $C D$ with the initial and boundary data functions $\bar{u}_{0}, \bar{f}$ (resp. $\underline{u}_{0}, \underline{f}$ ) which satisfy 
assumption (A). For any nonnegative $\chi \in C_{0}^{\infty}\left(Q_{T}\right)$ there exist positive constants $C_{1}$ and $C_{2}$ such that

$$
\begin{aligned}
\iint_{Q_{T}} r^{d}(u-\bar{u}) \chi d r d t \leq & C_{1} \int_{1}^{\infty} r^{d} e^{-r}\left(u_{0}(r)-\bar{u}_{0}(r)\right)^{+} d r \\
& +C_{2} \int_{0}^{T}\left(f^{m}(t)-\bar{f}^{m}(t)\right)^{+} d t \\
\left(\text { resp. } \iint_{Q_{T}} r^{d}(u-\underline{u}) \chi d r d t \leq\right. & C_{1} \int_{1}^{\infty} r^{d} e^{-r}\left(u_{0}(r)-\underline{u}_{0}(r)\right)^{+} d r \\
& \left.+C_{2} \int_{0}^{T}\left(f^{m}(t)-\underline{f}^{m}(t)\right)^{+} d t\right) .
\end{aligned}
$$

Corollary 4. Let $u$ and $\bar{u}$ (resp. $\underline{u}$ ) be as in Theorem 2. If $u_{0} \leq \bar{u}_{0}$ (resp. $\underline{u}_{0} \leq u_{0}$ ) a.e. in $(1, \infty)$ and $f \leq \bar{f}$ (resp. $\underline{f} \leq \bar{f}$ ) a.e. in $(0, T)$ then $u \leq \bar{u}$ (resp. $\underline{u} \leq u$ ) in $S_{T}$.

Corollary 5. Let $u$ and $\tilde{u}$ be the weak solutions of Problem CD with the data $u_{0}$, $f$ and $\tilde{u}_{0}, \tilde{f}$ respectively. If $u_{0} \leq \tilde{u}_{0}$ a.e. in $(1, \infty)$ and $f \leq \tilde{f}$ a.e. in $(0, T)$ then $u \leq \tilde{u}$ in $Q_{T}$.

Corollary 6. The weak solution of Problem CD is unique.

The proof of Theorem 2 goes essentially in the same way as the proof of Theorem 1 and we only indicate main differences. Instead of Lemma 1 we now use the following.

LEMma 3. A function $u$ is a weak solution of Problem CD if and only if $u$ satisfies instead of (1.3) the identity

$$
\begin{aligned}
& \iint_{P} r^{d}\left\{u \xi_{t}+u^{m} r^{-d}\left(r^{d} \xi_{r}\right)_{r}\right\} d r d t \\
& \quad+\int_{1}^{R} r^{d} u_{0}(r) \xi(r, 0) d r+\int_{0}^{T} f^{m}(t) \xi_{r}(1, t) d t-\left.\int_{0}^{T} r^{d} u^{m} \xi_{r} d t\right|_{r=R}=0
\end{aligned}
$$

for all rectangles $P \equiv[1, R] \times[0, T]$ with $1<R<\infty$ and for all nonnegative $\xi \in C^{2,1}(P)$ which vanish for $t=T, r=1$ and $r=R$. Similarly, defining a weak supersolution (resp. subsolution) of Problem $C D$ one should replace the equality in (2.16) by $\leq$ (resp. $\geq$ ).

Instead of problem (2.8)-(2.9) we consider now the problem

$$
\left\{\begin{array}{l}
\xi_{t}+a_{n} r^{-d}\left(r^{d} \xi_{r}\right)_{r}=-\chi \quad \text { in } P \\
\left.\xi\right|_{r=1}=\left.\xi\right|_{r=R}=\left.\xi\right|_{t=T}=0,
\end{array}\right.
$$

and the role of Lemma 2 is now played by

Lemma 4. There are positive constants $C_{1}, C_{2}, C_{3}$ and $C_{4}$ that do not depend on $n$ and $R$, and such that

$$
\begin{gathered}
0 \leq \xi^{n, R}(r, t) \leq C_{1} e^{-r} \quad \text { for }(r, t) \in P, \\
0 \leq \xi_{r}^{n, R}(1, t) \leq C_{2} \quad \text { for } t \in[0, T], \\
-C_{2} e^{-R} \leq \xi_{r}^{n, R}(R, t) \leq 0 \quad \text { for } t \in[0, T], \\
\iint_{P} a_{n}\left\{\left(r^{d} \xi_{r}^{n, R}\right)_{r}\right\}^{2} d r d t \leq C_{3} .
\end{gathered}
$$


We omit the proofs.

REMARK 1. All the results of this section extend to the equation

$$
u_{t}=r^{-d}\left(r^{d} \varphi(u)_{r}\right)_{r},
$$

where $\varphi \in C^{1}([0, \infty)), \varphi(0)=\varphi^{\prime}(0)=0$ and $\varphi$ is nondecreasing function on $[1, \infty)$.

3. Existence and regularity results. Let $D \subset \mathbb{R}_{+} \times(0, T]$ be a domain and let $u>0$ be a classical solution of equation (1.1) in D. Following [14] we define new dependent variables

$$
v=u^{m} \quad \text { and } \quad w=(m /(m-1)) u^{m-1} .
$$

We have

$$
\begin{gathered}
v_{r}=u w_{r}, \\
u_{r}=(1 / m) u^{2-m} w_{r},
\end{gathered}
$$

and

$$
\begin{gathered}
v_{t}=m v^{(m-1) / m}\left\{r^{-d}\left(r^{d} v_{r}\right)_{r}\right\}, \\
w_{t}=(m-1) w\left\{r^{-d}\left(r^{d} w_{r}\right)_{r}\right\}+w_{r}^{2}
\end{gathered}
$$

in $D$.

For any $0<\eta_{1}<\eta_{1}^{\prime}<\eta_{2}^{\prime}<\eta_{2}$ let us denote $R=\left(\eta_{1}, \eta_{2}\right) \times(0, T]$ and $R^{\prime}=$ $\left(\eta_{1}^{\prime}, \eta_{2}^{\prime}\right) \times(0, T]$. The following lemma is an extension of a well-known regularity result for the porous media equation in spatial dimension one [1].

Lemma 5. Let $w \in C^{3,1}(R), 0<w \leq M$ be a solution of equation (3.5) in $R$. Then for all $(r, t) \in R^{\prime}$ we have

$$
\left|w_{r}(r, t)\right| \leq K\left(\eta_{1}^{-2}+t^{-1}+1\right)^{1 / 2},
$$

where the constant $K$ depends only on $\eta_{1}^{\prime}-\eta, \eta_{2}-\eta_{2}^{\prime}, M, m$ and $d$. In addition, if $w, w_{r} \in C\left(\left(\eta_{1}, \eta_{2}\right) \times[0, T]\right)$ and $M_{1}=\sup _{\eta_{1}<r<\eta_{2}}\left|w_{r}(r, 0)\right|<\infty$ then for all $(r, t) \in R^{\prime}$ we have

$$
\left|w_{r}(r, t)\right| \leq K\left(\eta_{1}^{-2}+1\right)^{1 / 2},
$$

where now the constant $K$ depends on $\eta_{1}^{\prime}-\eta, \eta_{2}-\eta_{2}^{\prime}, M, M_{1}, m$ and $d$.

Proof. We apply the Bernstein technique in the form used in [1], [10] and [12].

Following [1] we define a new dependent variable $\widetilde{w}$ by $w=\Theta(\widetilde{w})$, where $\Theta(s):=$ $M s(3-s) / 2$ for all $s \in[0,1]$. Then $0<\widetilde{w}<1$ and $\widetilde{w}$ satisfies

$$
\widetilde{w}_{t}=(m-1) w\left[\widetilde{w}_{r r}+(d / r) \widetilde{w}_{r}\right]+\left[(m-1) w \frac{\Theta^{\prime \prime}}{\Theta^{\prime}}+\Theta^{\prime}\right] \widetilde{w}_{r}^{2}
$$

in $R$, where $\Theta^{\prime}$ and $\Theta^{\prime \prime}$ stand for $\Theta^{\prime}(\widetilde{w})$ and $\Theta^{\prime \prime}(\widetilde{w})$ respectively. Differentiating with respect to $r$ we find that $p=\widetilde{w}_{r}$ satisfies

$$
p_{t}-(m-1) w\left[p_{r r}+(d / r) p_{r}\right]=\Sigma_{1} p p_{r}+\Sigma_{2} p^{3}+\Sigma_{3} p^{2}+\Sigma_{4} p
$$

in $R$, with

$$
\begin{gathered}
\Sigma_{1}:=(m+1) \Theta^{\prime}+2(m-1) w\left(\Theta^{\prime \prime} / \Theta^{\prime}\right), \quad \Sigma_{2}:=m \Theta^{\prime \prime}+(m-1) w\left(\Theta^{\prime \prime} / \Theta^{\prime}\right)^{\prime}, \\
\Sigma_{3}:=(m-1)(d / r) \Theta^{\prime}, \quad \Sigma_{4}:=-(m-1) w\left(d / r^{2}\right) .
\end{gathered}
$$


For fixed $0<\tau \leq T$, let us denote $R_{\tau}=\left(\eta_{1}, \eta_{2}\right) \times(0, \tau]$ and $R_{\tau}^{\prime}=\left(\eta_{1}^{\prime}, \eta_{2}^{\prime}\right) \times(0, \tau]$. Following [10] we take the cut-off function $\xi$ of the form

$$
\xi(r, t)=\frac{t}{\tau} \chi(r)
$$

for $(r, t) \in \overline{R_{\tau}}$, where $\chi \in C^{2}\left(\left[\eta_{1}, \eta_{2}\right]\right)$ is such that $\chi\left(\eta_{1}\right)=\chi\left(\eta_{2}\right)=0,0 \leq \chi \leq 1$, and, $\chi(r)=1$ for all $r \in\left[\eta_{1}^{\prime}, \eta_{2}^{\prime}\right]$. Finally we consider the function $z=\xi^{2} p^{2}$ in $\overline{R_{\tau}}$. At the point $\left(r_{0}, t_{0}\right) \in R_{\tau}$ at which $z$ attains its maximum we have

$$
z_{r}=0 \quad \text { and } \quad z_{t}-(m-1) w z_{r r} \geq 0 .
$$

Performing differentiations in (3.9) and combining with (3.8) yields

$$
-\Sigma_{2} \xi^{2} p^{4} \leq\left(\Sigma_{3} \xi-\Sigma_{1} \xi_{r}\right) \xi p^{3}+\left[\xi \xi_{t}+(m-1) w\left(2 \xi_{r}^{2}+\Sigma_{5} \xi \xi_{r}-\xi \xi_{r r}\right)+\Sigma_{4} \xi^{2}\right] p^{2}
$$

at $\left(r_{0}, t_{0}\right)$, where $\Sigma_{5}=d / r$. Now observe that in view of the choice of $\Theta$

$$
(M / 2) \leq \Theta^{\prime} \leq(3 / 2) M, \quad \Theta^{\prime \prime}=-M, \quad\left|\Theta^{\prime \prime} / \Theta^{\prime}\right| \leq 2 \quad \text { and } \quad\left(\Theta^{\prime \prime} / \Theta^{\prime}\right)^{\prime} \leq-(4 / 9),
$$

and then $0<M \leq-\Sigma_{2}$ (that's the point!). Therefore there exist constants $K_{1}$ depending on $\eta_{1}, M, \max \left|\xi_{r}\right|, m$ and $d$, and $K_{2}$ which depends on the same parameters as well as $\max \left|\xi_{t}\right|=1 / \tau$ and $\max \left|\xi_{r r}\right|$, such that

$$
\xi^{2} p^{4} \leq K_{1} \xi|p|^{3}+K_{2} p^{2}
$$

at $\left(r_{0}, t_{0}\right)$. Applying the Cauchy inequality and recalling that $z \leq z\left(r_{0}, t_{0}\right)$ in $\overline{R_{\tau}}$ we obtain from (3.11) that

$$
z(r, t) \leq K_{1}^{2}+2 K_{2}
$$

for all $(r, t) \in \overline{R_{\tau}}$. Since $z=\widetilde{w}_{r}^{2}$ in $\overline{R_{\tau}^{\prime}}$ and taking into account how $K_{1}$ depends on $\eta_{1}$, and how $K_{2}$ depends on $\eta_{1}$ and $\tau$ this implies that

$$
\widetilde{w}_{r}^{2}(r, \tau) \leq K_{3}\left(\eta_{1}^{-2}+\tau^{-1}+1\right)
$$

for all $r \in\left[\eta_{1}^{\prime}, \eta_{2}^{\prime}\right]$, where the constant $K_{3}$ depends on $\eta_{1}^{\prime}-\eta, \eta_{2}-\eta_{2}^{\prime}, M, m$ and $d$. Noting that $w_{r}=\Theta^{\prime}(\widetilde{w}) \widetilde{w}_{r}$ this provides the first part of the theorem in view of the arbitrariness of $\tau$.

The proof of the second part of the lemma is similar. The main difference is that now we take $\xi(r, t)=\chi(r)$ for $(r, t) \in R$ with the same function $\chi$ as above. We omit the details.

3.1. Cauchy-Neumann problem. For the Problem $\mathrm{CN}$ the following result holds.

Theorem 3. There exists a weak solution u of Problem CN. Moreover,

(i) $u$ is a classical solution of equation (1.1) in a neighbourhood of any point $\left(r_{0}, t_{0}\right) \in$ $Q_{T}$ where $u\left(r_{0}, t_{0}\right)>0$;

(ii) the derivative $\left(u^{m}\right)_{r}$ exists and is continuous in $Q_{T}$ and, if $\delta>1$ then

$$
\left|\left(u^{m}\right)_{r}(r, t)\right| \leq K\left(t^{-1}+1\right)^{1 / 2} u(r, t)
$$

for all $(r, t) \in Q_{\delta, T}=[\delta, \infty) \times(0, T]$, where the constant $K$ depends on $\delta,\left\|u_{0}\right\|_{\infty},\|g\|_{\infty}$, $m$ and $d$, and does not depend on $T$. 
(iii) if $1<m<2$ then the derivative $u_{r}$ exists and is continuous in $Q_{T}$ and, if $\delta>1$ then

$$
\left|u_{r}(r, t)\right| \leq K\left(t^{-1}+1\right)^{1 / 2} u^{2-m}(r, t)
$$

for all $(r, t) \in Q_{\delta, T}$, where the constant $K$ depends on $\delta,\left\|u_{0}\right\|_{\infty},\|g\|_{\infty}, m$ and $d$, and does not depend on $T$.

Proof. We adapt the classical method introduced in [14] and construct a weak solution of Problem CN as a pointwise limit of a sequence of strictly positive classical solutions of equation (1.1).

1. Existence of the solution. Denote $L=\max \left(\left\|u_{0}\right\|_{\infty},\|g\|_{\infty}\right)$ and define for each integer $n>n_{0}=\max \left(4, T^{-1}\right)$

$$
\begin{aligned}
u_{0, n} & =\widetilde{u}_{0, n} * \omega_{n}+1 / n, \\
g_{n} & =\widetilde{g}_{n} * \omega_{n}+1 / n,
\end{aligned}
$$

where

$$
\widetilde{u}_{0, n}(r)= \begin{cases}u_{0}(r) & \text { for } r \in(1+1 / n, n-2), \\ L & \text { for } r \in[n-2, \infty), \\ \{(L+1 / n)(r-1+1 / n)\}^{1 / m} & \text { for } r \in[1-1 / n, 1+1 / n], \\ 0 & \text { otherwise, }\end{cases}
$$

and

$$
\widetilde{g}_{n}(t)= \begin{cases}g(t) & \text { for } t \in(1 / n, T] \\ L & \text { for } t \in[-1 / n, 1 / n] \\ 0 & \text { otherwise, }\end{cases}
$$

and $\left\{\omega_{n}\right\}_{n=1}^{\infty}$ is the standard sequence of mollifiers with $\operatorname{supp} \omega_{n} \subset[-1 / n, 1 / n]$. It is easy to verify that

(i) $u_{0, n} \in C^{\infty}([1, \infty)), \quad g_{n} \in C^{\infty}([0, T]$,

(ii) $1 / n \leq u_{0, n} \leq L+(1 / n), \quad 1 / n \leq g_{n} \leq L+(1 / n)$,

(iii) $\left(u_{0, n}^{m}\right)^{\prime}(1)=-g_{n}(0)$,

(iv) $u_{0, n} \rightarrow u_{0}$ a.e. in $(1, \infty)$ and $g_{n} \rightarrow g$ a.e. in $(0, T)$ as $n \rightarrow \infty$.

Now we shall work with $v$ variable defined in (3.1). For each integer $n>n_{0}$ consider the problem

$$
\begin{cases}v_{t}=\Phi_{n}(v)\left\{r^{-d}\left(r^{d} v_{r}\right)_{r}\right\} & \text { in } Q_{n}=(1, n) \times(0, T], \\ v(r, 0)=u_{0, n}^{m}(r) & \text { for } r \in[1, n], \\ v_{r}(1, t)=-g_{n}(t) & \text { for } t \in[0, T], \\ v(n, t)=(L+(1 / n))^{m} & \text { for } t \in[0, T],\end{cases}
$$

where $\Phi_{n}(s)$ is a sufficiently smooth function on $\mathbb{R}$ such that $\Phi_{n}(s)=m s^{(m-1) / m}$ if $(1 / n)^{m} \leq s \leq M_{n}$, and that $0<(1 / 2) \Phi_{n}(1 / n) \leq \Phi_{n}(s) \leq 2 \Phi\left(M_{n}\right)$ if $s \in \mathbb{R}$, with a constant $M_{n}$ to be specified later. It follows from the classical theory of uniformly 
parabolic equations [13] that there exists a unique solution $v_{n} \in C^{2,1}\left(\bar{Q}_{n}\right)$ of problem (3.16)-(3.19). We show that $v_{n}$ is a solution of (3.4) for a proper choice of the constant $M_{n}$. First note that by the maximum principle argument as used in the proof of the left hand inequality in $(2.10)$,

$$
(1 / n)^{m} \leq \min _{Q_{n}} v_{n}
$$

Note also that equation (3.16) has obvious stationary solutions of the form $\bar{v}(r)=A r^{1-d}+$ $B$ if $d \neq 1$ and $\bar{v}(r)=A \ln r+B$ if $d=1$, with $A, B \in \mathbb{R}$. One can choose constants $A=A(d, L)$ and $B=B(d, L)$ if $d>1$, and $A=A(d, L)$ and $B=B(d, L, n)$ if $d \leq 1$, such that

$$
\begin{gathered}
\left(v_{n}\right)_{r}(1, t) \geq \bar{v}^{\prime}(1) \text { for } t \in[0, T], \\
v_{n}(r, 0) \leq \bar{v}(r) \text { for } r \in[1, n], \\
v_{n}(n, t) \leq \bar{v}(n) \text { for } t \in[0, T]
\end{gathered}
$$

(we leave the details for the reader). Then by a maximum principle argument $v_{n} \leq \bar{v}$ and consequently

$$
\max _{Q_{n}} v_{n} \leq \max _{[1, n]} \bar{v}=: M_{n} .
$$

Note that if $d>1$ then $\max _{[1, n]} \bar{v} \equiv M$ does not depend on $n$ (still depends on $L, m$ and $d$ ). With this choice of $M_{n}, v_{n}$ is a solution of (3.16). A standard argument (cf. [6]) shows that $\left(v_{n}\right)_{r} \in C^{2,1}\left(Q_{n}\right)$ and then, by (3.1) we have also $\left(u_{n}^{m-1}\right)_{r} \in C^{2,1}\left(Q_{n}\right)$.

We show that the sequence $\left\{v_{n}\right\}_{n>n_{0}}$ is bounded from above uniformly with respect to $n$ also when $d \leq 1$. In view of the maximum principle it is enough to show this in the case when the boundary data function $g_{n} \equiv L+1$. For this we adapt the argument from [14]. Define

$$
\omega_{n}(r)=h_{n}(r)((r / n)+L+2)
$$

for $n>n_{0}$ and $r \in[0, n]$, where $h_{n}$ are smooth functions on $[1, n]$ and such that

$$
\begin{gathered}
h_{n}(1)=h_{n}(n)=0, \quad h_{n}^{\prime}(1)=1, \\
h_{n}(r)>0 \text { for } r \in[1, n], \\
h_{n},\left|h_{n}^{\prime}\right|,\left|h_{n}^{\prime \prime}\right| \leq \text { const (uniformly with respect to } n \text { ), }
\end{gathered}
$$

and consider functions

$$
y_{n}=\left(v_{n}+\omega_{n}\right) \exp \{-\kappa t\}
$$

for some $\kappa>0$. We have $\left(\omega_{n}\right)^{\prime}(1)>L+2$, whence $\left(y_{n}\right)_{r}(1, t)>\exp \{-\kappa t\}>0$ and consequently $y_{n}$ cannot attain its maximum at $r=1$. If this maximum is attained at the remaining part of parabolic boundary of $Q_{n}$ then clearly its value is bounded from above uniformly with respect to $n$ by the bound which depends on $L$ and the upper bound of $h_{n}$. Now consider the case when the maximum of $y_{n}$ is attained in $Q_{n}$. By (3.4) and (3.20) we have

$$
m v_{n}^{(m-1) / m}\left\{r^{-d}\left(r^{d}\left(y_{n}\right)_{r}\right)_{r}\right\}-\kappa y_{n}-\left(y_{n}\right)_{t}=m v_{n}^{(m-1) / m}\left\{r^{-d}\left(r^{d}\left(\omega_{n}\right)^{\prime}\right)^{\prime}\right\} \exp \{-\kappa t\} .
$$

At the maximum point of $y_{n}$ we have $r^{-d}\left(r^{d}\left(y_{n}\right)_{r}\right)_{r}-\left(y_{n}\right)_{t} \leq 0$ and hence

$$
\kappa y_{n}+m v_{n}^{(m-1) / m}\left\{r^{-d}\left(r^{d}\left(\omega_{n}\right)^{\prime}\right)^{\prime}\right\} \exp \{-\kappa t\} \leq 0
$$


at that point. This inequality implies in turn that

$$
v_{n} \leq\left\{-(m / \kappa)\left[r^{-d}\left(r^{d}\left(\omega_{n}\right)^{\prime}\right)^{\prime}\right]\right\}^{m}
$$

at the maximum point of $y_{n}$. Then necessarily the values of $y_{n}$ at that point are bounded from above uniformly with respect to $n$. Consequently, $y_{n}$ are bounded from above uniformly with respect to $n$ on $Q_{n}$ and then the same is true for $v_{n}$. Note that by (3.20) and (3.21), having fixed $\kappa$ and functions $\omega_{n}$ this upper bound depends on $L, m$ and $d$.

Now we return to considering the functions $u_{n}=\left(v_{n}\right)^{1 / m}$. Let $1<\delta$ and $0<\tau<T$ be fixed. According to Lemma 5

$$
\left|\left(u_{n}^{m-1}\right)_{r}(r, t)\right| \leq K\left(t^{-1}+2\right)^{1 / 2} \quad \text { on } Q_{n-1} \cap Q_{\delta, T},
$$

where the constant $K$ depends on $\delta, L, m$ and $d$, and does not depend on $T$ and $n$. Hence, by the result of [5] there exists a positive constant $C$ independent of $n(C=$ $C(\delta, \tau, T, L, m, d))$ and such that

$$
\left|u_{n}^{m-1}(r, t)-u_{n}^{m-1}\left(r^{\prime}, t^{\prime}\right)\right| \leq C\left(\left|r-r^{\prime}\right|+\left|t-t^{\prime}\right|^{1 / 2}\right)
$$

for all $(r, t),\left(r^{\prime}, t^{\prime}\right) \in Q_{n-2} \cap[\delta, \infty] \times[\tau, T]$. Therefore, in view of arbitrariness of $\delta$ and $\tau,\left\{u_{n}^{m-1}\right\}_{n>n_{0}}$ are equicontinuous on any compact subset of $Q_{T}$ and clearly the same is true for $\left\{u_{n}\right\}_{n>n_{0}}$. Now we can apply the Ascoli-Arzela Theorem and a diagonalization argument to conclude that there exist a subsequence $n_{k} \rightarrow \infty$ and a function $u \in C\left(Q_{T}\right)$ such that $u_{n_{k}} \rightarrow u$ as $k \rightarrow \infty$ uniformly on compact subsets of $Q_{T}$. It is easy to verify that $u$ is a weak solution of Problem CN. Moreover, uniqueness of the weak solution implies that $u_{n} \rightarrow u$ as $n \rightarrow \infty$ uniformly on compacts of $Q_{T}$.

2. Regularity. The proof of (i) is standard: if $\left(r_{0}, t_{0}\right) \in Q_{T}$ and $u\left(r_{0}, t_{0}\right)>0$ then there exist $\gamma>0$ and a neighbourhood $N_{0} \subset Q_{T}$ of $\left(r_{0}, t_{0}\right)$ such that $u_{n} \geq \gamma$ in $N_{0}$ for all $n>n_{0}$. In turn it follows from (3.23) that $\left\|u_{n}^{m-1}\right\|_{C^{0+\alpha}\left(N_{0}\right)}$ (for any $\alpha \in(0,1)$ ) may be estimated independently of $n$. Considering once more the functions $\left\{v_{n}\right\}_{n>n_{0}}$ and applying the linear regularity theory [4] for equation (3.4) we can find a neighbourhood $N_{1} \subset N_{0}$ of $\left(r_{0}, t_{0}\right)$ such that $\left\|v_{n}\right\|_{C^{2+\alpha}\left(\overline{N_{1}}\right)}$ is bounded uniformly with respect to $n$. This implies that $u$ is a classical solution of equation (1.1) in $N_{1}$.

Now we turn to the proof of (ii). First note that by the preceding argument the derivative $\left(u^{m}\right)_{r}(r, t)$ exists and is continuous in a neighbourhood of any point $(r, t) \in Q_{T}$ at which $u(r, t)>0$. Especially $\left(u^{m}\right)_{r}(r, t)=\lim _{n \rightarrow \infty}\left(u_{n}^{m}\right)_{r}(r, t)$ provided $u(r, t)>0$.

Fix now $\delta>1$. It follows from (3.2) and (3.22) that there exists a constant still denoted by $K, K=K(\delta, T, L, m, d)$ and such that

$$
\left|\left(u_{n}^{m}\right)_{r}(r, t)\right| \leq K\left(t^{-1}+1\right)^{1 / 2} u_{n}(r, t)
$$

for all $(r, t) \in Q_{n} \cap Q_{\delta, T}$ and $n>n_{0}$. This implies that $\lim _{n \rightarrow \infty}\left(u_{n}^{m}\right)_{r}(r, t)=0$ if $u(r, t)=0$. Thus $\Phi(r, t)=\lim _{n \rightarrow \infty}\left(u_{n}^{m}\right)_{r}(r, t)$ exists for all $(r, t) \in Q_{T}$ and by $(3.24)$

$$
|\Phi(r, t)| \leq K\left(t^{-1}+1\right)^{1 / 2} u(r, t)
$$

for all $(r, t) \in Q_{\delta, T}$. This in turn implies that $\Phi(r, t)$ is a continuous function on $Q_{T}$. In order to end up the proof of (3.14) it remains to show that $\Phi\left(r_{0}, t_{0}\right)=\left(u^{m}\right)_{r}\left(r_{0}, t_{0}\right)$ for 
all $\left(r_{0}, t_{0}\right) \in Q_{T}$ at which $u\left(r_{0}, t_{0}\right)=0$. But this an immediate consequence of

$$
u^{m}\left(r, t_{0}\right)=\int_{r_{0}}^{r} \Phi(x, t) d x
$$

which holds for all $\left(r, t_{0}\right) \in Q_{T}$, and which we justify by applying (3.24) and the Lebesgue dominated convergence theorem to

$$
u_{n}^{m}\left(r, t_{0}\right)-u_{n}^{m}\left(r_{0}, t_{0}\right)=\int_{r_{0}}^{r}\left(u_{n}^{m}\right)_{r}(x, t) d x .
$$

For the proof of (iii) we repeat the argument used in the proof of (ii) applying (3.3) instead of (3.2).

REMARK 2. It follows from (3.23) that if $\delta>1$ and $0<\tau<T$ then

$$
\left|u(r, t)-u\left(r^{\prime}, t^{\prime}\right)\right| \leq C\left(\left|r-r^{\prime}\right|+\left|t-t^{\prime}\right|^{1 / 2}\right)^{\nu}
$$

for all $(r, t),\left(r^{\prime}, t^{\prime}\right) \in[\delta, \infty) \times[\tau, T]$, where $\nu=\min (1,1 /(m-1))$, with $C=C(\delta, \tau, T$, $\left.\left\|u_{0}\right\|_{\infty},\|g\|_{\infty}, m, d\right)$.

REMARK 3. If $u_{0}^{m-1}$ is uniformly Lipschitz continuous on $[1, \infty)$ then

(i) for any $\delta>1$,

$$
\left|\left(u^{m}\right)_{r}(r, t)\right| \leq K u(r, t)
$$

for all $(r, t) \in Q_{\delta, T}$, where the constant $K$ depends on $\delta,\left\|u_{0}\right\|_{\infty},\|g\|_{\infty}, m$, $d$, and a Lipschitz constant of $u_{0}^{m-1}$, and does not depend on $T$.

(ii) if $1<m<2$ then for any $\delta>1$,

$$
\left|u_{r}(r, t)\right| \leq K u^{2-m}(r, t)
$$

for all $(r, t) \in Q_{\delta, T}$, where the constant $K$ depends on $\delta,\left\|u_{0}\right\|_{\infty},\|g\|_{\infty}, m, d$, and a Lipschitz constant of $u_{0}^{m-1}$, and does not depend on $T$. This is a consequence of (3.7) and properties of the sequence $\left\{u_{0, n}\right\}$.

Concerning regularity up to the boundary $t=0$ the following result holds.

Theorem 4. Let $u$ be the weak solution of Problem $C N$. If $r_{0} \in(1, \infty)$ and $\operatorname{ess} \lim _{r \rightarrow r_{0}} u_{0}(r)=l$ then $\lim _{(r, t) \rightarrow\left(r_{0}, 0\right)} u(r, t)=l$.

Proof. The result can be proved by recalling the construction process given in the proof of Theorem 3 and adapting the the standard barrier method [4, 6, 15].

3.2. Cauchy-Dirichlet problem. Corresponding results for the Problem CD read as follows.

Theorem 5. There exists a weak solution u of Problem CD. Moreover,

(i) $u$ is a classical solution of equation (1.1) in a neighbourhood of any point $\left(r_{0}, t_{0}\right) \in$ $Q_{T}$ where $u\left(r_{0}, t_{0}\right)>0$;

(ii) the derivative $\left(u^{m}\right)_{r}$ exists and is continuous in $Q_{T}$ and, if $\delta>1$ then

$$
\left|\left(u^{m}\right)_{r}(r, t)\right| \leq K\left(t^{-1}+1\right)^{1 / 2} u(r, t)
$$

for all $(r, t) \in Q_{\delta, T}=[\delta, \infty) \times(0, T]$, where the constant $K$ depends on $\delta,\left\|u_{0}\right\|_{\infty},\|f\|_{\infty}$, $m$ and $d$, and does not depend on $T$. 
(iii) if $1<m<2$ then the derivative $u_{r}$ exists and is continuous in $Q_{T}$ and, if $\delta>1$ then

$$
\left|u_{r}(r, t)\right| \leq K\left(t^{-1}+1\right)^{1 / 2} u^{2-m}(r, t)
$$

for all $(r, t) \in Q_{\delta, T}$, where the constant $K$ depends on $\delta,\left\|u_{0}\right\|_{\infty},\|f\|_{\infty}, m$ and $d$, and does not depend on $T$.

Proof. The proof follows strategy of the proof of Theorem 3: a weak solution of Problem $\mathrm{CD}$ is constructed as a pointwise limit of a sequence of strictly positive classical solutions of equation (1.1). We omit the details.

REMARK 4. The statements of Remark 3 remain true for the solution of Problem CD with the obvious replacement $\|g\|_{\infty}$ by $\|f\|_{\infty}$.

Regularity of the solution at the points of parabolic boundary is given by the following theorem.

Theorem 6. Let $u$ be the weak solution of Problem CD.

(i) If $r_{0} \in(1, \infty)$ and ess- $\lim _{r \rightarrow r_{0}} u_{0}(r)=l$ then $\lim _{(r, t) \rightarrow\left(r_{0}, 0\right)} u(r, t)=l$.

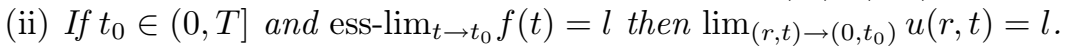

(iii) If $\operatorname{ess}_{-} \lim _{r \rightarrow 1+} u_{0}(r)=\operatorname{ess}_{-} \lim _{t \rightarrow 0+} f(t)=l$ then $\lim _{(r, t) \rightarrow(1,0)} u(r, t)=l$.

Proof. The proof uses the barrier method technique.

REMARK 5. The results of this section extend to the equation (2.23), where $\varphi$ satisfies

(i) $\varphi \in C^{1}([0, \infty)) \cap C^{2+\alpha}((0, \infty))$ for some $\alpha \in(0,1], \varphi(0)=\varphi^{\prime}(0)=0$ and $\varphi^{\prime \prime}(s)>0$ for $s>0$,

(ii) $\int_{0}^{1} \frac{\varphi^{\prime}(s)}{s} d s<\infty$,

(iii) $\lim _{s \rightarrow \infty} \frac{\varphi^{\prime}(s)}{\varphi(s)}=0$.

\section{References}

[1] D. G. Aronson, Regularity properties of flows through porous media, SIAM J. Appl. Math. 17 (1969), 461-467.

[2] D. G. Aronson, The porous medium equation, in Nonlinear Diffusion Problems, A. Fasano and M. Primicerio (eds.), Lecture Notes in Mathematics 1224, Springer-Verlag, Berlin, 1986, 1-46.

[3] J. I. Diaz and R. Kersner, On a nonlinear parabolic equation in infiltration or evaporation through a porous medium, J. Differential Equations 69 (1987), 368-403.

[4] A. Friedman, Partial Differential Equations of Parabolic Type, Prentice-Hall, Englewood Cliffs, New Jersey, 1964.

[5] B. H. Gilding, Hölder continuity of solutions of parabolic equations, J. London Math. Soc. 13 (1976), 103-106.

[6] B. H. Gilding, A nonlinear degenerate parabolic equation, Ann. Scuola Norm. Pisa 4 (1977), 393-432. 
[7] B. H. Gilding, Improved theory for a nonlinear degenerate parabolic equation, Ann. Scuola Norm. Pisa 16 (1989), 165-224.

[8] B. H. Gilding and J. Goncerzewicz, Localization of solutions of exterior domain problems for the porous media equation with radial symmetry, SIAM J. Math. Anal. 31 (2000), 862-893.

[9] B. H. Gilding and J. Goncerzewicz, Large-time behaviour of solutions of the exteriordomain Cauchy-Dirichlet problem for the porous media equation with homogeneous boundary data, Monatsh. Math. 150 (2007), 11-39.

[10] M. A. Herrero and J. L. Vázquez, On the one-dimensional nonlinear heat equation with absorption: regularity of solutions and interfaces, SIAM J. Math. Anal. 18 (1987), 149-167.

[11] A. S. Kalashnikov, The Cauchy problem in a class of growing functions for equations of unsteady filtration type, Vestnik Mosk. Univ. Ser. VI Mat. Mekh. 6 (1963), 17-27 (in Russian).

[12] A. S. Kalashnikov, Some problems of the qualitative theory of non-linear degenerate second-order parabolic equations, Russian Math. Surveys 42 (1987), no. 2, 169-222 (in Russian); English transl.: Uspekhi Mat. Nauk 42 (1987), no. 2, 135-176.

[13] O. A. Ladyzhenskaya, V. A. Solonnikov and N. N. Ural'ceva, Linear and Quasilinear Equations of Parabolic Type, Translations of Mathematical Monographs 23, Amer. Math. Soc., Providence, RI. 1968.

[14] O. A. Oleinik, A. S. Kalashnikov and Y.-L. Chzhou, The Cauchy problem and boundary problems for equations of the type of non-stationary filtration, Izv. Akad. Nauk SSSR Ser. Mat. 22 (1958), 667-704 (in Russian).

[15] O. A. Oleinik and T. D. Ventzell, The first boundary value problem and the Cauchy problem for quasilinear parabolic equations, Mat. Sb. 41 (1957), 105-128 (in Russian).

[16] L. A. Peletier, The porous media equation, in: Applications of Nonlinear Analysis in the Physical Sciences, H. Amann et al. (eds.), Pitman Advanced Publishing Program, Boston, 1981, 229-241.

[17] M. H. Protter and H. F. Weinberger, Maximum Principles in Differential Equations, Prentice-Hall, Englewood Cliffs, New Jersey, 1967.

[18] J. L. Vázquez, An introduction to the mathematical theory of the porous medium equation, in: Shape Optimization and Free Boundaries M. C. Delfour and G. Sabidussi (eds.), Kluwer, Dordrecht, 1992, 347-389.

[19] J. L. Vázquez, The Porous Medium Equation. Mathematical Theory, Oxford University Press, 2006. 
